\title{
Subaru AO Coronagraphic and Direct Imaging of YSOs
}

\author{
Motohide Tamura ${ }^{1}$, Misato Fukagawa ${ }^{2}$, Masahiko Hayashi $^{3}$, and \\ SDPS/CIAO teams
}

\begin{abstract}
A cold near-infrared stellar coronagraph combined with adaptive optics (CIAO) is introduced. As an open-use instrument on the Subaru 8.2-m telescope, it has been used for several star formation studies with high spatial resolutions (from natural seeing of about 0.6 arcsec down to 0.07 arcsec with adaptive optics). A brief explanation is described of the instrument as well as its current main project of systematic surveys of disks and young companions around $\mathrm{T}$ Tauri stars and Herbig Ae/Be stars. In particular, observations of HL Tau are presented in some details. Our images of HL Tau show several new circumstellar features including the presence of a red $H-K$ color region of $\sim 150 \mathrm{AU}$, probably corresponding to the small circumstellar disk. The observations of a high density stellar cluster of MWC 137 are also reported. It appears to be a cluster of very low-mass stars around Herbig Be star or a cluster of B stars around a supergiant.
\end{abstract}

\section{What is CIAO?}

The Japanese 8.2-m Subaru telescope is equipped with a unique near-infrared (NIR) instrument, CIAO (Coronagraphic Imager with Adaptive Optics). CIAO is a combination of relatively low-order (36-elements) adaptive optics and the classical Lyot-type coronagraph working at NIR wavelengths. Although the halo reduction is limited by the current adaptive optics performance, it is very suitable for observations of objects in the close vicinity of bright objects with a high spatial resolution ( $\sim 0 .^{\prime \prime} 07$ at wavelength of $2.2 \mu \mathrm{m}$ ) because of its clean PSF (point-spread-function) and availability of small occulting masks ( $0 .{ }^{\prime \prime} 1$ in diameter or larger).

CIAO is optimized at NIR wavelengths $(1-5 \mu \mathrm{m})$ where the adaptive optics works most efficiently and the effect of scattering by the telescope and instrument optics is smaller. Optical components for coronagraph, occulting masks and Lyot stops, are all cooled, and various sizes and shapes of masks and stops are available. CIAO is equipped with the standard broad band filters $\left(z J H K K s L^{\prime} M^{\prime}\right)$ as well as a number of narrow band filters. Slit spectroscopy

\footnotetext{
${ }^{1}$ National Astronomical Observatory of Japan, 2-21-1 Osawa, Mitaka, Tokyo 181-8588, Japan

${ }^{2}$ The University of Tokyo, 7-3-1 Hongo, Bunkyo-ku, Tokyo 113-0033, Japan

${ }^{3}$ Subaru Telescope, National Astronomical Observatory of Japan, 650 North A'ohoku Place, Hilo, HI 96720, USA
} 
with a grism of several hundred resolutions is available. Polarimetry is also available both with and without adaptive optics. CIAO utilizes one $1024 \times 1024$ InSb detector array, covering a field-of-view of either $22^{\prime \prime}$ or $11^{\prime \prime}$. The instrument achieved its first light on the Subaru telescope in 2000 February and its first combination with the Subaru adaptive optics was made in 2001 January. It has been under open use since 2001 October. More details of the instrument can be found elsewhere (Tamura et al. 1998; Tamura et al. 2000; Tamura et al. 2003; Murakawa et al. 2003).

We are currently conducting systematic searches for circumstellar disks and young planets around nearby star forming regions under a part of the Subaru Observatory Project (SDPS; Subaru Disk and Planet Searches) and other various projects. By last year typically $0 .{ }^{\prime \prime} 1$ imaging of a few dozens of T Tauri stars (TTSs) and Herbig Ae/Be (HAEBE) stars at $H$ band has been conducted. Because of the proximity of these objects and the small mask size, it enables us to observe companions at a few tens of AU from a central star.

Several disk images with Subaru/CIAO have already been published. One of the most clear circumbinary disks (rings) around GG Tau has been reported by Itoh et al. (2002). An original disk around a Herbig Ae star, HD 150193A, has been discovered by Fukagawa et al. (2003). HD 150193 is in fact a Herbig Ae star - T Tauri star pair, and only the primary is found to be associated with a disk with their detection limit. More details of the survey of HAEBE stars are presented by Fukagawa in this meeting (IAU00968).

We also found several point-like sources within our field-of-view $\left(\sim 22^{\prime \prime}\right)$ around the target TTSs and HAEBE stars. These candidate companions are planned to be followed up by checking a common proper motion or by NIR spectroscopy.

\section{HL Tau}

\subsection{Observations and Results}

HL Tau is unusual among TTSs. It is the source of a collimated optical jet at PA $=51^{\circ}$. HL Tau has the highest optical polarization of $11 \%$ among TTSs, and 2 $\mu \mathrm{m}$ polarization of $0.8 \%$. It is a strong IRAS source, having a flat spectral energy distribution out to $100 \mu \mathrm{m}$. In the millimeter and submillimeter continuum, it is by far the brightest source among all TTSs in Taurus. Its circumstellar mass is estimated to be near $0.1 \mathrm{M}_{\odot}$. A flattened distribution of ${ }^{13} \mathrm{CO}$ molecular emission extending to radii of $2000 \mathrm{AU}$ is delineated and the densest part of the disk is much smaller $\left(0 .^{\prime \prime} 7=100 \mathrm{AU}\right)$.

The observations of HL Tau at $H$ and $K$ bands were conducted in 2002 November with CIAO mounted on the Subaru telescope. CIAO can usually use either the central star of TTS or a nearby star for the adaptive optics guide star. In the observations of HL Tau we used the nearby XZ Tau as a guide star. The resolution of these observations is $0.2^{\prime \prime}$ without any deconvolutions. The coronagraph mode was not used in this observation.

Our $H$ band image overlaid with the HST optical image (Stapelfeldt et al. 1995; S95) is shown in Fig. 1. Registration was made on the assumption that a faint point like knot in the optical image corresponds to the NIR point- 


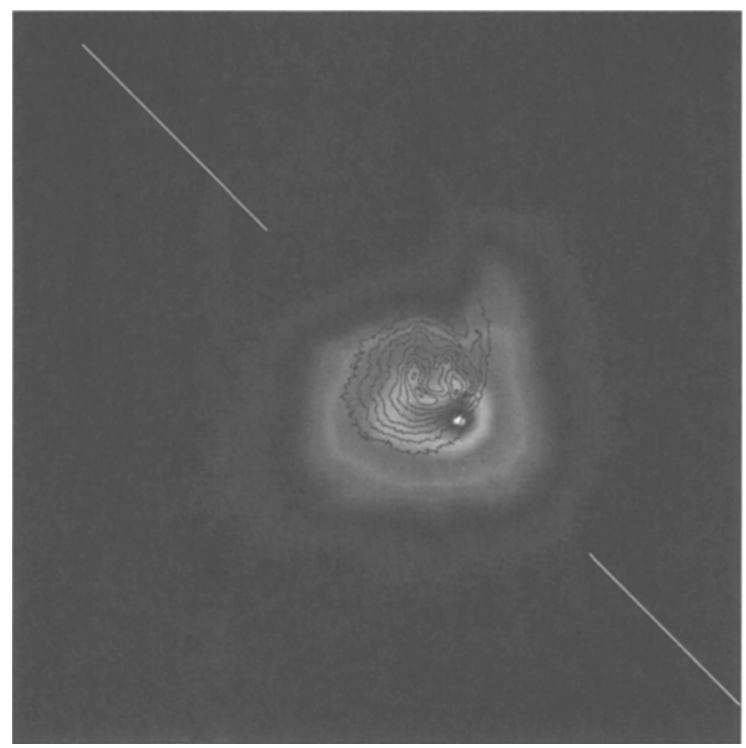

Figure 1. Subaru/CIAO $H$ band image of HL Tau overlaid on the HST optical (F555W) image. The field-of-view is $11^{\prime \prime} \times 11^{\prime \prime}$. The direction of the optical jets is shown in two lines.

like source. This was also justified by our cross-correlation with various other features. There are a number of known and new features seen in this figure as well as in our $K$ band image, as summarized below: (1) Central unresolved source whose peaks at $H$ and $K$ bands are coincident with each other, as suggested by Close et al. (1997). (2) Inner halo, where most of the NIR light comes from. This part extends to a bright knot $\sim 1^{\prime \prime}$ to the east. The knot is more prominent at $H$ than at $K$, thus introducing an apparent shift of the peak positions from $K$ to $H$ (and $J$ ) in the previous low resolution studies (see Weintraub et al. 1995). (3) Outer halo, which includes the following features; (3a) flipped-C shape nebula (see S95), (3b) broad extension to the east, (3c) two arms extending to the north, the outer arm only seen at NIR and the inner arm seen at both optical and NIR, (3d) disk shadow seen as a "waist" of the larger nebula (3e) running at a $\mathrm{PA}=135^{\circ}$. (Such a feature is commonly seen in several protostar sources in Taurus, Tamura et al. 1991), and (3e) on the $20^{\prime \prime}$ scale, the nebula looks cometary, heading toward the SW. Both (2) and (3) are of reflection origin (e.g., Lucas et al. 2003).

\subsection{Discussion}

Comparison with optical and NIR nebulae: two cavities By comparing optical and NIR nebulae, we can obtain the insight on the origin of both nebulae. There are several similarities between the NIR and optical nebulae. Their inner parts both appear clumpy and lack the outflow symmetry. Most of the nebular light originates in a flipped-C shaped ridge of emission. Such an (NIR) morphology is occasionally seen in massive YSOs with outflows, although their size is much 


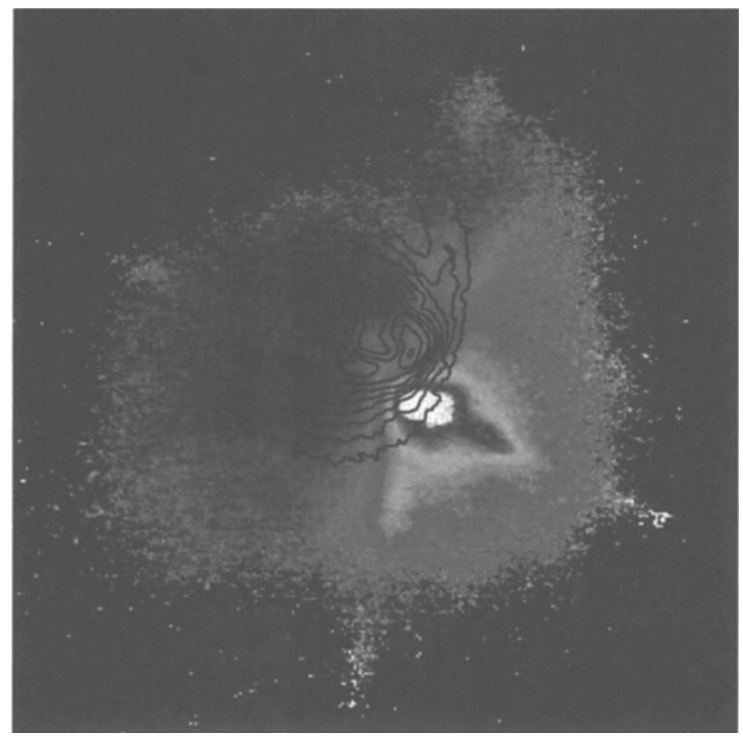

Figure 2. Subaru/CIAO $H-K$ color image overlaid on the HST optical image. The field-of-view is $\sim 6^{\prime \prime} \times 6^{\prime \prime} . H-K$ spans from 0.4 to 2.5 mag. Note that the four spiders of the Subaru telescope make an artifact of higher $H-K$ color.

larger (e.g., GGD 27, GL 2591). There is also some difference between the NIR and optical nebulae, however. Both the optical size of the flipped-C and the larger envelope is smaller than their NIR size.

In summary, there are two kinds of cavities; the smaller one corresponds to (3a) and the larger one consists of (3c) and (3b). In both cavities, their inner part is optically brighter and their outer part is brighter at NIR wavelengths. Therefore, both cavities become optically thinner toward their axes. The large scale optical jet (Mundt et al. 1988) is misaligned with the axis of the inner cavity, although the axis of the optical jet at small scale (in the small cavity) is not yet well determined (Ray et al. 1996). We speculate that the two cavities suggest episodic outflows.

$H-K$ image: Evidence for inner disk Although there have been several radio observations suggesting the presence of a small scale ( $100 \mathrm{AU})$ disk around HL Tau, no direct evidence for HL Tau's circumstellar disk is seen in the WFPC2 images. Close et al. (1997) claimed the detection of such a small disk directly seen in their adaptive optics images. However, it is not clear in our new images, although our images show a disk shadow of $\sim 500$ AU scale (3d). This shadow could be due to the presence of small scale disk near the central unresolved core.

Fig. 2 shows $H-K$ image from our data. The image clearly shows a large scale gradient of $H-K$ color along $\mathrm{PA}=150^{\circ}$ over the entire nebula ( $1000 \mathrm{AU})$, well correlated with the optical nebula. This is mostly due to the large envelope observed in ${ }^{13} \mathrm{CO}$ (Sargent \& Beckwith 1987, 1991; Hayashi et al. 1993). In addition, there is a local peak of $H-K$ color around the central 

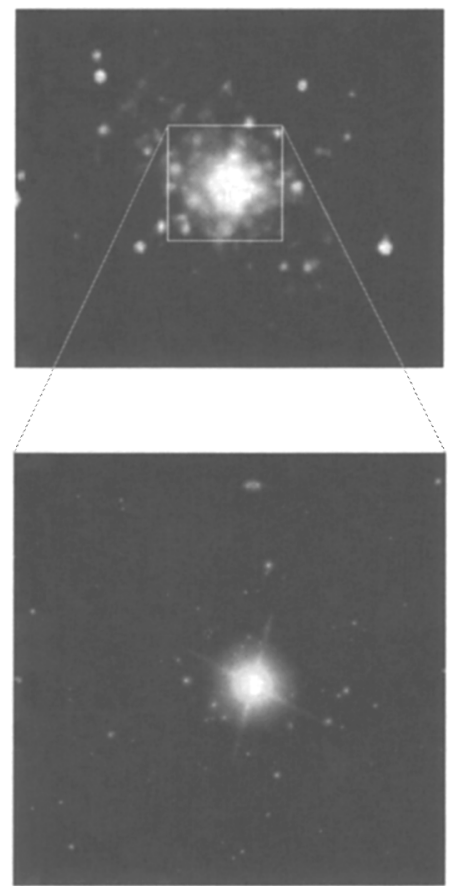

Figure 3. Comparison of SIRIUS (above) and CIAO (below) data of MWC 137. The field-of-view of the CIAO image is $22^{\prime \prime} \times 22^{\prime \prime}$.

core extending to the west with a diameter of $\sim 150 \mathrm{AU}$. We believe that this is due to the small scale disk, although its morphology is not disk-like in our $H-K$ color image. Its size is comparable to that of the $2 \mathrm{~mm}$ continuum disk of NMA (Kitamura et al. 2002) and $2.7 \mathrm{~mm}$ continuum disk of BIMA (Mundy et al. 1996; Looney et al. 2000).

\section{3. $\quad$ MWC 137}

MWC 137 is a HAEBE star of spectral type B0 at a distance of $1.3 \mathrm{kpc}$ (Hillenbrand et al. 1992), associated with very strong lines of $\mathrm{H} \alpha$ and $\mathrm{HeI}$ and other numerous lines as well as photometric variabilities. It is a millimeter continuum source of extended emission (Henning et al. 1998). It is associated with an HII region, S2-266. Recently, it has been claimed to be a $\mathrm{B}[\mathrm{e}]$ supergiant with a peculiar ring-like nebula at a distance of $>6 \mathrm{kpc}$ (Esteban and Fernández 1998). In order to study this interesting source, we have conducted (1) wide-field $\left(\sim 8^{\prime}\right)$ $J H K s$ simultaneous imaging with SIRIUS (Nagayama et al. 2002) on the IRSF 1.4-m telescope in South Africa, and (2) high resolution ( 0."1) $K$ imaging with Subaru/CIAO. We have detected $\sim 460$ sources within $\mathrm{r}=216^{\prime \prime}$ with SIRIUS (12 $<K s<16)$ and $\sim 190$ sources within $\mathrm{r}=11^{\prime \prime}$ with CIAO $(13<K<20)$. Both data (Fig. 3) clearly show the presence of a star cluster originally discovered by Testi et al. (1997). 
The most interesting thing is the high stellar density of this cluster revealed by the CIAO image. Its density is greater than $10^{4}$ stars $\mathrm{pc}^{-2}$, assuming a distance of $1.3 \mathrm{kpc}$. The background stars contribute less than $10 \%$, estimated from both extrapolation from the SIRIUS data and from galactic star count model. Such a high density cluster is not known for any HAEBE stars. The population of this cluster is worth noting; its luminosity function is dominated by faint sources $(K>16)$, which correspond to young brown dwarfs if the age of the cluster is $1 \mathrm{Myr}$ and the distance is $1.3 \mathrm{kpc}$. In this case, the cluster around MWC 137 is a high density cluster with very low-mass stars. Even if MWC 137 is a supergiant at $9.4 \mathrm{kpc}$, the cluster is composed of more than 100 B0-B5 (ZAMS) stars. Such a high density B star cluster around a supergiant is also very unique in our Galaxy. Therefore, further detailed studies on this source are desirable.

\section{References}

Close, L. M. et al. 1997, ApJ, 478, 766.

Esteban, C., \& Fernández, M. 1998, MNRAS, 298, 185.

Fukagawa, M. et al. 2003, ApJ, 590, L49.

Henning, Th. et al. 1998, A\&A, 336, 565 .

Hayashi, M., Ohashi, N., \& Miyama, S. M. 1993, ApJ, 418, L71.

Hillenbrand, L. A. et al. 1992, ApJ, 397, 613.

Itoh, Y. et al. 2002, PASJ, 54, 963.

Kitamura, Y. et al. 2002, ApJ, 581, 357.

Looney, L. W., Mundy, L. G., \& Welch, W. J. 2000, ApJ, 529, 477.

Lucas, P. W., Fukagawa, M. et al. 2003, in preparation.

Mundt, R., Ray, T. P., \& Buhrke, T. 1988, ApJ, 333, L69.

Mundy, L. G. et al. 1996, ApJ, 464, L169.

Murawaka, K. et al. 2003, Proc. SPIE, 4841, 881.

Nagayama, T. et al. 2002, Proc. SPIE, 4841, 2002.

Ray, T. P. et al. 1996, ApJ, 468, L103.

Sargent, A. I., \& Beckwith, S. 1987, ApJ, 323, 294.

Sargent, A. I., \& Beckwith, S. V. W. 1991, ApJ, 382, L31.

Stapelfeldt, K. R. et al. 1995, ApJ, 449, 888.

Tamura, M., Gatley, I., Waller, W., \& Werner, M. W. 1991, ApJ, 378, 611.

Tamura, M. et al. 1998, Proc. SPIE, 3354, 845.

Tamura, M. et al. 2003, Proc. SPIE, 4843, 190.

Tamura, M. et al. 2000, Proc. SPIE, 4008, 1153.

Testi, L. et al. 1997, A\&A, 320, 159.

Weintraub, D. A., Kastner, J. H., \& Whitney, B. A. 1995, ApJ, 452, L141. 


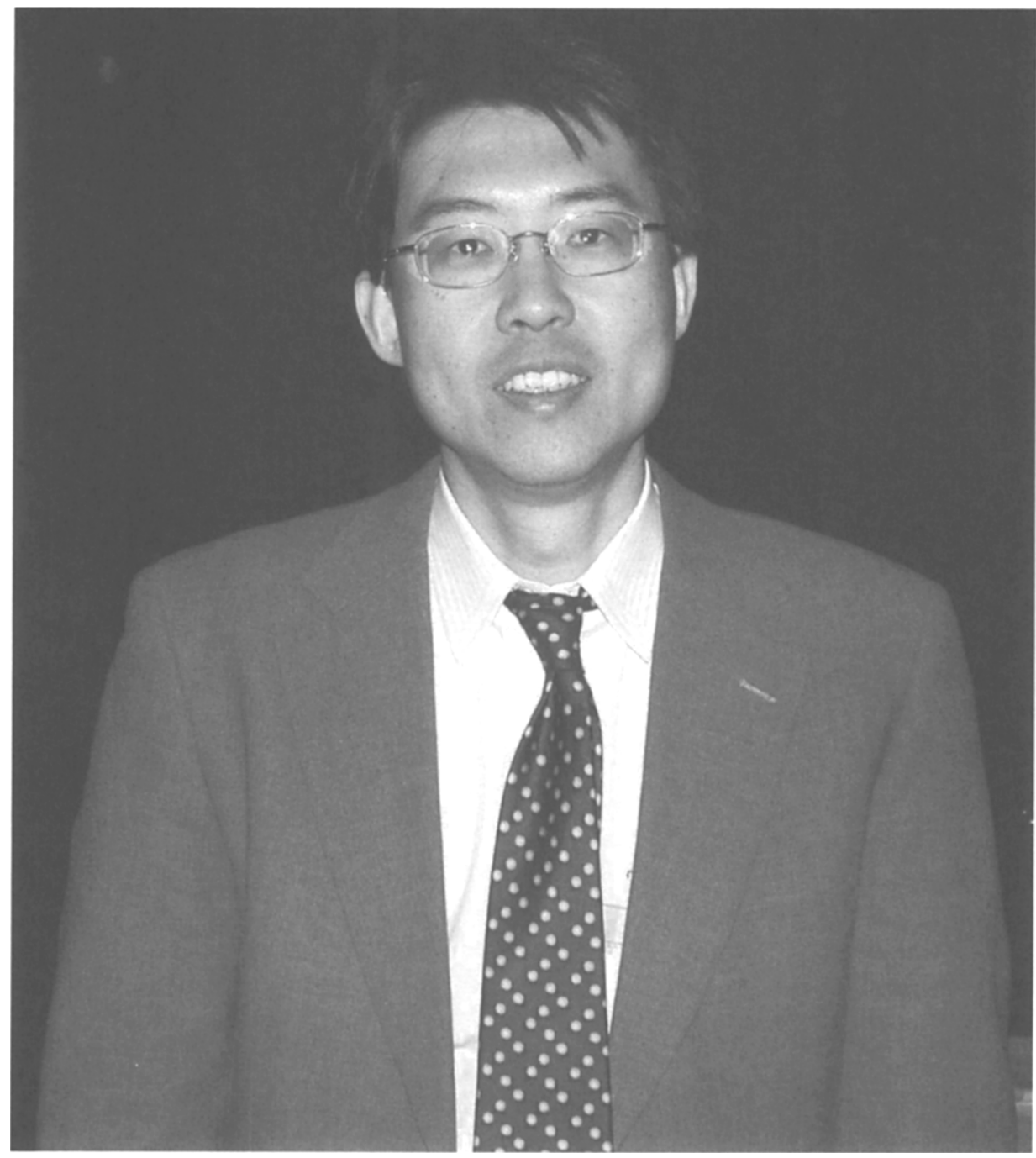

Motohide Tamura 